\title{
Patient Treatment Preferences for Osteoporosis
}

\author{
Liana Fraenkel, MD, MPH [Associate Professor of Medicine] ${ }^{1,2}$, Barbara Gulanski, MD, MPH \\ [Associate Professor of Medicine] $^{1,2}$, and Dick Wittink, PhD [Professor of Marketing] \\ 1 VA Connecticut Healthcare System \\ 2 Yale University School of Medicine
}

\section{Abstract}

Background-Adherence with osteoporosis treatment is poor. Incorporation of patient treatment preferences into decision-making may improve patient adherence. The objective of this study was to examine patient preferences for available and promising treatment options in osteoporosis.

Methods-We recruited patients who had recently (within 2 weeks) undergone bone densitometry and were found to have osteoporosis. Consenting participants completed an Adaptive Conjoint Analysis questionnaire to determine their treatment preferences for oral bisphosphonates taken once per week, intravenous bisphosphonates given every three months, intravenous bisphosphonates given once per year, and subcutaneous rhPTH. We performed simulations based on respondents' values for route of administration, absolute reduction in risk of vertebral and hip fractures over five years, and risk of adverse effects to predict each individual respondent's treatment choice.

Results-The study sample included 201 women and 11 men (median age =73). Patients' treatment preferences were strongly influenced by route of administration. Patients' preferred treatment option, across all simulations, was bisphosphonates. Among treatment naive participants $(\mathrm{N}=80), 52(65 \%)$ preferred an annual infusion over oral weekly bisphosphonates. Participants with poorer perceived health status, those with a high perceived risk of future fracture, as well as participants preferring to treat health problems without doctors or prescription drugs were more likely to prefer an annual infusion over weekly pills.

Conclusions-Patient preferences for osteoporosis treatment options are strongly influence by route of administration. Because of this, despite the added benefits of rhPTH, patients' preferred treatment option for osteoporosis is bisphosphonates. Amongst those preferring bisphosphonates, many preferred annual infusions over weekly oral medications. This finding emphasizes the need to incorporate individual patient preferences into treatment decisions for osteoporosis. The latter is especially important given the poor rates of long-term adherence with osteoporosis medications.

\section{Keywords}

Osteoporosis; Bisphosphonates; Recombinant Human Parathyroid Hormone; Decision-Making

In the U.S. today, 10 million Americans have osteoporosis, and 50\% of women and 25\% of men will have an osteoporotic fracture their lifetime ${ }^{(1)}$. The estimated national direct expenditure for osteoporotic fractures is over 47 million dollars per day. More importantly, osteoporotic fractures result in significant functional impairment, decreased quality of life, and

Please correspond with: Liana Fraenkel, MD, MPH, Yale University School of Medicine, Section of Rheumatology, 300 Cedar St,

TAC\#525, P.O. Box 208031, New Haven, CT 06520-8031 E-mail:liana.fraenkel@yale.edu.

*Dr. Wittink is deceased.

This study was funded by the Arthritis Foundation Clinical Science Grant. Dr. Fraenkel is also supported by the K23 Award AR048826-01 A1. This work was supported by the NIH/NCRR/GCRC Program Grant \#M01-RR00125. 
increased mortality ${ }^{(2)}$. The impact of this major public health problem is expected to increase exponentially as the population ages.

Measures used to reduce bone loss, including calcium and vitamin D supplementation, regular weight-bearing exercise, and avoidance of tobacco and excessive alcohol, have essentially no risk and are generally recommended for all patients. Currently available prescription medications to treat osteoporosis include bisphosphonates, calcitonin, hormonal replacement therapy, raloxifene, and teriparatide (human recombinant parathyroid hormone, rhPTH).

Results from the Women's Health Initiative, however, raise concerns regarding the long-term use of hormonal replacement therapy as a first line agent for treatment of osteoporosis (2). Similarly, the role of raloxifene will be better elucidated when results from the STAR (The Study of Tamoxifen and Raloxifene) and RUTH (Raloxifene Use for the Heart) trials are available.

Alendronate and risedronate are two oral bisphosphonates approved by the Food and Drug Administration for the treatment of osteoporosis which are now available as a single tablet taken once weekly. Both have been shown to decrease the risk of vertebral and hip fractures in large randomized controlled trials ${ }^{(3-5)}$. Ibandronate, a monthly oral bisphosphonate has also been recently been approved for the treatment of osteoporosis. Intravenous bisphosphonates administered every three months decrease bone loss, but data demonstrating their ability to decrease fracture rates is lacking ${ }^{(6,7)}$. Zoledronic acid, a more potent intravenous bisphosphonate approved for the treatment of malignant hypercalcemia, was recently shown to have the same effect on bone turnover and bone density as oral bisphosphonates when administered once yearly ${ }^{(8)}$.

rhPTH, unlike the anti-resorptive agents, exerts its protective effect, in part, by stimulating bone growth. Despite producing increased bone turnover rates, rhPTH increases bone mineral density and decreases the risk of osteoporotic fractures by approximately $60 \%{ }^{(9)}$. Potential drawbacks associated with PTH include its route of administration (daily subcutaneous injections), high cost, and unknown long-term safety-profile.

Because of the numbers of persons affected, the public health impact of treating osteoporosis is enormous. However, at the individual patient level many patients will not derive any benefit from treatment. For example, assuming the 10 year probability of sustaining a hip fracture for 75 year old women with osteoporosis is $22 \%$ (10); if 100 such women all took alendronate, 11 fractures would be prevented and 11 fractures would occur despite treatment. Thus, of 100 women taking treatment, 89 derive no benefit ${ }^{(11)}$.

Each individual patient's preference for treatment will therefore depend on how he or she weighs the risk of future morbidity and possible mortality over the uncertain risk of long-term toxicity, bothersome adverse effects, and costs related to treatment. Consequently, treatment decisions should be based on physician expertise and explicitly derived patient preferences. This process of decision-making not only adheres to the principles of informed consent and patient autonomy, but also has been shown to increase patient satisfaction and improve compliance ${ }^{(12-14)}$, both of which are essential to ensure successful long-term treatment of osteoporosis and ultimately prevent fractures.

To the best of our knowledge, studies have not quantified patient preferences for prevention of osteoporotic fractures. Understanding patient preferences for prevention of osteoporotic fractures is especially important given that adherence to these medications is poor ${ }^{(15)}$. The objective of this study was to examine patient treatment preferences for available and promising treatment options in osteoporosis using an interactive computerized questionnaire which elicits preferences based on how patients make trade-offs between the risks and benefits of the treatment options under consideration. 


\section{METHODS}

\section{Participants}

We recruited postmenopausal women and men (over the age of 65) who had recently (within 2 weeks) undergone bone densitometry. Participants were drawn from six centers performing bone densitometry in the greater New Haven area.

All patients undergoing bone densitometry, who were able to understand English, were asked whether they agreed to be contacted by a research assistant to learn more about, and potentially participate in, a study examining patients' opinions about medications for osteoporosis.

Patients were asked to fill out a form indicating whether or not they wished to be contacted.

Persons with osteoporosis [T score (at total hip or lumbar spine) at or below -2.5 ] and/or a Fracture Index Score $\geq 6^{(16)}$ (the cutoff at which evaluation for treatment is recommended ${ }^{(16)}$ ) were eligible to participate. These criteria were chosen to ensure that all participants in the study would be considered eligible for pharmacologic treatment of osteoporosis. Only patients with known secondary causes of osteoporosis, esophagitis, severe heartburn, the inability to sit upright for at least 30 minutes, or previous allergic reactions to bisphosphonates or calcitonin (ascertained by self-report) were excluded. These criteria were meant to exclude patients for whom one or more of the treatment options might not be medically reasonable choices. The research protocol was approved by the HIC committee at our institution.

\section{Preference Measurement}

Participants first underwent a standardized educational session with the research assistant to briefly explain the pathophysiology of osteoporosis and its complications. The information presented to participants was based on patient information materials published by the National Osteoporosis Foundation (see Appendix 1). The educational session was performed to ensure that all participants had the same information available to them before performing the preference task.

Consenting participants completed an Adaptive Conjoint Analysis (ACA) questionnaire to determine their treatment preferences. This approach has been previously described in detail ${ }^{(17-22)}$. Briefly, ACA collects and analyzes preference data using an interactive computer program. Preferences are derived by asking respondents to make trade-offs between the characteristics of competing treatment options under consideration rather than explicitly naming these options. ACA assumes that each treatment option is a composite of different characteristics. The characteristics included in this study were: route of administration (pill taken once a week, intravenous infusion given over two hours every three months, intravenous infusion given over 15 minutes once a year, daily subcutaneous injection), absolute risk reduction of vertebral fractures over five years, absolute risk reduction of hip fractures over five years, and risk of adverse effects (gastrointestinal adverse effects and infusion reactions). Risk of sarcoma was not included in the questionnaire.

Medication characteristics were first explained in detail to participants using lay terminology and then, because of space constraints, presented in abbreviated format in the computerized questionnaire. In this study, the ACA survey contained two sets of questions. First, respondents were asked to rate the importance of the difference between the highest and lowest estimate of each characteristic on a four point scale, thereby allowing ACA to learn enough about each respondent's values to construct initial utility estimates. In this context "utility" is a number that represents the value a respondent associates with a particular characteristic, with higher utilities indicating increased value. An example of this type of question is provided in Appendix 2a. In the second set of questions respondents evaluated a series of paired concepts. Each 
question involved choosing one option from a pair in which one is superior in one characteristic and the opposing option is superior in the other (see Appendix 2b). ACA constructs pairs by examining all the possible ways the characteristics can be combined and then chooses pairs of options with similar utilities for which it expects respondents to be indifferent (based on previous responses). The program uses the information obtained from each paired comparison to update the estimates of each respondent's utilities and to select the next pair of options. Final utilities are generated using regression analysis ${ }^{(21)}$.

We created two versions of the ACA questionnaire (high fracture risk: Fracture Index $>7$, lower fracture risk: Fracture Index $=6$ or $7^{(16)}$, so that each participant was presented with individualized probability estimates. Estimates of risk were obtained using the Fracture Index scoring system developed by Black et al ${ }^{(16)}$.

\section{Covariates}

All covariates were collected by self report. Overall health status was measured using a global health status question: "In general would you say your health is: "Excellent", "Very good", "Good", "Fair" or "Poor" (23). Attitudes towards utilization of medical services were ascertained using relevant questions from the Medical Care Preference Scale (24): "I prefer to treat most health problems without help from doctors or prescription drugs" and "For most health problems I would rather take a prescription drug than a non prescription drug" coded on a five item response scale ranging from "Strongly disagree" to "Strongly agree". Participants stating that they "Agreed" or "Strongly agreed" were classified as preferring to treat health problems without doctors or prescription drugs and preferring prescription over non prescription drugs for the first and second statements respectively. Perceived risk of future fracture over five years was coded on a five item response scale:"1 in 100 people like me", "5 in 100 people like me", "10 in 100 people like me", "20 in 100 people like me", and "30 in 100 people like me will have an osteoporotic fracture in the next five years". Responses of "10 in 100 people like me" or more were coded as having a high perceived risk of fracture. Worry about developing a stooped posture and hip fracture were each evaluated using a seven item response scale ranging from "None of the time" to "All of the time". Respondents stating that they were worried about either becoming stooped or having a hip fracture "A good bit of the time" or more were classified as being worried about future fractures.

\section{Analyses}

Preference data derived from ACA (version 3.0, Sawtooth Software, Inc., Sequim, WA) were imported into SAS and merged with the patient characteristics data set. We calculated the relative importances of the characteristics studied by dividing the range of each characteristic (utility of highest estimate - utility of lowest estimate) by the sum of ranges of all characteristics, and multiplying it by 100 . The relative importances reflect how much influence each treatment characteristic has on respondents' choices.

We performed simulations to predict each individual respondent's treatment choice. For each simulation, ACA predicts preferences based on the utilities derived from the conjoint questionnaire using least squares regression analysis. Note that the participant does not evaluate treatment alternatives directly. Rather, the participant considers the trade-offs between conflicting characteristics. Answers to carefully selected patient-specific questions allow the investigator to infer values for specific treatment characteristics. These values are then used to predict which option most closely suits each patient's individual priorities. Preferences were predicted for oral bisphosphonates taken once per week, intravenous bisphosphonates given every three months, intravenous bisphosphonates given once per year, and subcutaneous rhPTH. Although not approved for the treatment of osteoporosis, bisphosphonate infusions are available and were included in this study to determine their impact on patient preferences. Once 
monthly oral bisphosphonates were not included because this option was not available at the time of this study. Calcitonin was not included because this choice is dominated by the other options included in the model (i.e. studies suggest that calcitonin is not as effective as bisphosphonates and like the latter may be associated with side effects).

In the base-case scenario, we described all bisphosphonates (regardless of route of administration) as being associated with a 50\% reduction in risk of future vertebral and hip fractures. rhPTH was described as conferring a $65 \%$ decreased risk of future vertebral fractures and a $50 \%$ decreased risk of future hip fractures. Although, the Neer trial ${ }^{(9)}$ did not have the power to examine hip fractures, we chose $50 \%$ risk reduction for this outcome based on the assumption that rhPTH would be at least as effective as bisphosphonates in reducing hip fractures. We then performed sensitivity analyses to examine how increasing efficacy or risk of adverse effects altered participants' preferences.

Associations between respondent characteristics and treatment preferences were examined using t-test and chi-square or Fisher's exact tests for continuous and categorical covariates respectively. Multivariate analyses were subsequently performed using multiple logistic regression.

\section{RESULTS}

\section{Participant Characteristics}

The study sample included 201 women and 11 men; median age 73 (range 46 to 90). Of the 233 patients agreeing to be contacted, 217 were eligible, of whom, 212 agreed to participate. The majority (87\%) of the sample was white, $50 \%$ were married; and $68 \%$ had at least some college education. One hundred seventy-three (82\%) subjects knew of bisphosphonates as a possible treatment option for osteoporosis, one was familiar with $\mathrm{rhPH}$, and other than this one participant, none had talked about using an injectable medication with their physician prior to the study. Participant characteristics are further described in Table 1.

\section{Relative Importance of Treatment Characteristics}

Figure 1 displays the relative importance of each medication characteristic on participants' treatment choices. Patients' treatment preferences were strongly influenced by route of administration. Prevention of vertebral fractures was felt to be as important, by the patients participating in the study, as prevention of hip fractures.

\section{Participants' Treatment Preferences}

Participants' treatment preferences are described in Table 2. For the base-case scenario, preferences were strongest and equally distributed between oral and intravenous bisphosphonates. Three percent of respondents were willing to use rhPTH over bisphosphonates in this scenario, and preferences changed little when the benefits of rhPTH were broadened to include increased effectiveness over bisphosphonates in preventing both vertebral and hip fractures. The distribution of treatment preferences did not differ by history of fracture or level of worry regarding future fracture (Table 3).

When oral bisphosphonates and rhPTH were described as not being associated with an increased risk of dyspepsia or nausea (to reflect equivalent rates of these adverse effects in both experimental and control groups in randomized controlled trials), the former becomes the preferred choice amongst all options studied. 


\section{Preference for Annual Infusions versus Oral Medications}

Among treatment naïve participants $(\mathrm{N}=80), 52(65 \%)$ preferred an annual infusion over oral weekly bisphosphonates. Treatment preferences for annual infusions versus weekly oral medication were not associated with age, education or prescription drug coverage (Table 4). We did not evaluate the association of gender or race with treatment preferences because of inadequate numbers of men and minority participants. In bivariate analyses, participants with poorer perceived health status, those with a high perceived risk of future fracture, as well as participants preferring to treat health problems without doctors or prescription drugs were more likely to prefer an annual infusion over weekly pills (Table 4). Health status and attitude towards doctors and prescription medicines remained positively associated with preference for annual infusions in a multivariate model (Table 4).

Among respondents currently using bisphosphonates, only attitude towards use of prescription drugs was associated with treatment preference for oral versus intravenous medication. More respondents choosing oral bisphosphonates preferred to treat most health problems with prescription drugs compared to $31 \%$ of respondents choosing the intravenous option (51\% versus $31 \%, \mathrm{p}=0.05$ ).

\section{DISCUSSION}

In this study, despite the added benefit of rhPTH, participants preferred bisphosphonates for the treatment of osteoporosis. While prevention of fractures was important in participants' decision-making, the added benefit conferred by PTH was not sufficient to overcome participants' dislike of daily subcutaneous injections. In addition, although associated with less morbidity and mortality, respondents in this study felt that prevention of vertebral fractures was as important as prevent of hip fractures. This may be due to fear of developing a cosmetic deformity, i.e. kyphosis.

Despite an overall strong preference for bisphosphonates, we found considerable variation in individual respondent's treatment preferences for weekly oral medication versus annual infusions. Variability in preferences was not related to sociodemographic characteristics; however, participants with poorer self reported health status as well as those with a high perceived risk of fracture were more likely to prefer an annual infusion, suggesting that patients might view infusions as being more effective than oral medications even when they are described as being associated with the same outcomes. The association between perceived fracture risk and preference for annual infusions did not reach statistical significance. This may have been due to the small number of participants in the multivariate analysis. Participants preferring to treat health problems without doctors or prescription drugs were also more likely to prefer annual infusions over weekly oral bisphosphonates. This finding suggests that an annual infusion should be discussed with patients who prefer to minimize utilization of traditional healthcare resources.

Strengths of this study include the methods used to elicit preferences. First, outcome data was individualized based on individual respondent's risk factors. Second, respondents' preferences were predicted based on how they made trade-offs between medication characteristics and therefore were not biased by recognition of specific treatment options. Careful evaluation of salient trade-offs is considered an essential component of high quality decision-making (25). Third, numerous studies have demonstrated that ACA produces internally consistent responses, and that it is a reliable and valid method of measuring preferences $(19-22,26)$. Moreover, use of interactive computer questionnaires minimizes interviewer bias, increases participants' interest and involvement in the task, and ensures that respondents evaluate all characteristics under consideration. 
In view of the known difficulties associated with communicating probabilistic information, we facilitated understanding of risk magnitude by providing both numerical estimates (natural frequencies) as well as graphical representations of probability data ${ }^{(27-29)}$. In addition, we provided outcomes for treated as well as untreated patients (see example Appendix $2 \mathrm{~b}$ ) and we used the same denominator throughout the survey ${ }^{(29)}$.

Our results must be interpreted in view of the limitations of this study. We could not include all medication characteristics or co-pays, since this would have overly complicated the questionnaire. For the same reason, we described bisphosphonates as being associated with the same risk and benefits, even though fracture data is not equivalent, or not available, for all types of bisphosphonates (e.g. zolendronic acid). Description of route of administration and adverse effects were abbreviated to ensure readability at a fifth grade level. Although, full disclosure of all details is ideal, patients' willingness to engage in making trade-offs decreases when the task is too difficult. For this reason we chose the most salient details for each medication characteristic. Inclusion of additional adverse effects (such as the theoretical possibility of osteogenic sarcoma) and cost, however, would be expected to further widen the gap between patient preferences for bisphosphonates over rhPTH.

Many of the participants interviewed were already on treatment for osteoporosis, because we could not recruit sufficient numbers of treatment naïve persons. The results in this study may therefore have been influenced by current medication use. In addition, most participants were Caucasian, female, and well-educated, thereby limiting the generalizability of the results.

In summary, we found that patient preferences for osteoporosis treatment options were strongly influence by route of administration. Because of this, despite the added benefits of rhPTH, patients' preferred treatment option for osteoporosis is bisphosphonates. Amongst those preferring bisphosphonates, many preferred annual infusions over weekly oral medications. This finding emphasizes the need to incorporate individual patient preferences into treatment decisions for osteoporosis. The latter is especially important given the poor rates of long-term adherence with osteoporosis medications.

\section{Acknowledgements}

We would like to thank all participants for their time and effort. Dr. Fraenkel had full access to all the data and takes full responsibility for the integrity of the data and the accuracy of the analysis. There are no potential conflicts or overlap with other publications to the best of our knowledge. The authors do not have any financial interests that would be considered a conflict of interest.

\section{References}

1. Black D. Report From the Fifth International Symposium on Recent Clinical Advances in Osteoporosis, International Symposium on Recent Clinical Advances in Osteoporosis, Honolulu, Hawaii. 2002.

2. Cooper C. The crippling consequences of fractures and their impact on quality of life. Am J Med 1997;103(2A):12S-19S. [PubMed: 9302893]

3. Black DM, Cummings SR, Karpf DB, et al. Randomised trial of effect of alendronate on risk of fracture in women with existing vertebral fractures. Fracture Intervention Trial Research Group. Lancet 1996;348:1535-41. [PubMed: 8950879]

4. Cummings SR, Black DM, Thompson DE, et al. Effect of alendronate on risk of fracture in women with low bone density but without vertebral fractures: results from the Fracture Intervention Trial. JAMA 1998;280:2077-82. [PubMed: 9875874]

5. Ensrud KE, Black DM, Palermo L, et al. Treatment with alendronate prevents fractures in women at highest risk: results from the Fracture Intervention Trial. Arch Intern Med 1997;157:2617-24. [PubMed: 9531231] 
6. Body JJ, Gaich GA, Scheele WH, et al. A randomized double-blind trial to compare the efficacy of teriparatide [recombinant human parathyroid hormone (1-34)] with alendronate in postmenopausal women with osteoporosis. J Clin Endocrinol Metab 2002;87:4528-35. [PubMed: 12364430]

7. Thiebaud D, Burckhardt P, Kriegbaum H, et al. Three monthly intravenous injections of ibandronate in the treatment of postmenopausal osteoporosis. Am J Med 1997;103:298-307. [PubMed: 9382122]

8. Reid IR, Brown JP, Burckhardt P, et al. Intravenous zoledronic acid in postmenopausal women with low bone mineral density. N Engl J Med 2002;346:653-61. [PubMed: 11870242]

9. Neer RM, Arnaud CD, Zanchetta JR, et al. Effect of parathyroid hormone (1-34) on fractures and bone mineral density in postmenopausal women with osteoporosis. N Engl J Med 2001;344:1434-41. [PubMed: 11346808]

10. Kannis JA, Johnell O, Oden A, Dawson A, De Laet C, Jonsson B. Ten year probabilities of osteoporotic fractures according to BMD and diagnostic thresholds. Osteoporos Int 2001;12:98995. [PubMed: 11846333]

11. Seeman E. Osteoporosis: trials and tribulations. Am J Med 1997;103:74S-87S. [PubMed: 9302899]

12. Greenfield S, Kaplan S, Ware JE Jr. Expanding patient involvement in care. Effects on patient outcomes. Ann Intern Med 1985;102:520-8. [PubMed: 3977198]

13. Edworthy SM, Devins GM. Improving medication adherence though patient education distinguishing between appropriate and inappropriate utilization. J Rheumatol 1999;26:1793-1801. [PubMed: 10451079]

14. Daltroy LH. Doctor-patient communication in rheumatological disorders. Baillieres Clin Rheumatol 1993;7:221-39. [PubMed: 8334710]

15. Solomon DH, Avorn J, Katz JN, et al. Compliance with osteoporosis medication. Arch Intern Med 2005;165:2414-19. [PubMed: 16287772]

16. Black DM, Steinbuch M, Palermo L, et al. An assessment tool for predicting fracture risk in postmenopausal women. Osteoporosis Int 2001;12:519-28.

17. Fraenkel L, Bogardus ST, Concato J, Felson DT, Wittink DR. Patient preferences for treatment of rheumatoid arthritis. Ann Rheum Dis 2004;63:1372-78. [PubMed: 15020312]

18. Fraenkel L, Bogardus ST, Felson DT, Wittink DR. Treatment options in knee osteoarthritis: the patient's perspective. Arch Intern Med 2004;164:1299-1304. [PubMed: 15226163]

19. Fraenkel L, Bodardus S, Wittink DR. Understanding patient preferences for the treatment of lupus nephritis with adaptive conjoint analysis. Med Care 2001;39:1203-16. [PubMed: 11606874]

20. Johnson RM. Adaptive Conjoint Analysis. Sawtooth Software Conference Proceedings 1987:25365.

21. Wittink DR, Bergenstuen T. Forecasting with conjoint analysis. In: Armstrong JS, ed. Principles of forecasting: a handbook for researchers and practitioners. Norwell, MA: Kluwer Academic Publishers: 2001.

22. Ryan M, Farrar S. Using conjoint analysis to elicit preferences for health care. BMJ 2000;320:153033. [PubMed: 10834905]

23. Ware JE, Snow KK, Kosinski M, Gandek B. SF-36 health status survey manual. Boston: The Health Institute, New England Medical Center 1993.

24. Ganther JM, Wiederholt JB, Kreling DH. Measuring patients' medical care preferences: care seeking versus self-treating. Med Decis Making 2001;21:133-40. [PubMed: 11310946]

25. Janis IL, Mann L. Decision-making. A psychological analysis of conflict, choice, and commitment. New York: The Free Press; 1985.

26. Green PE, Srinivasan V. Conjoint analysis in marketing: new developments with implications for research and practice. J Marketing 1990;54:3-17.

27. Braddock CH 3rd, Edwards KA, Hasenberg NM, Laidley TL, Levinson W. Informed decision making in outpatient practice: time to get back to basics [see comments]. Jama 1999;282:2313-20. [PubMed: 10612318]

28. Rothman AJ, Kiviniemi MT. Treating people with information: an analysis and review of approaches to communicating health risk information. Monogr Natl Cancer Inst 1999;25:44-51. [PubMed: 10854457]

29. Gigerenzer G. Calculated Risks New York: Simon \& Schuster; 2002. 


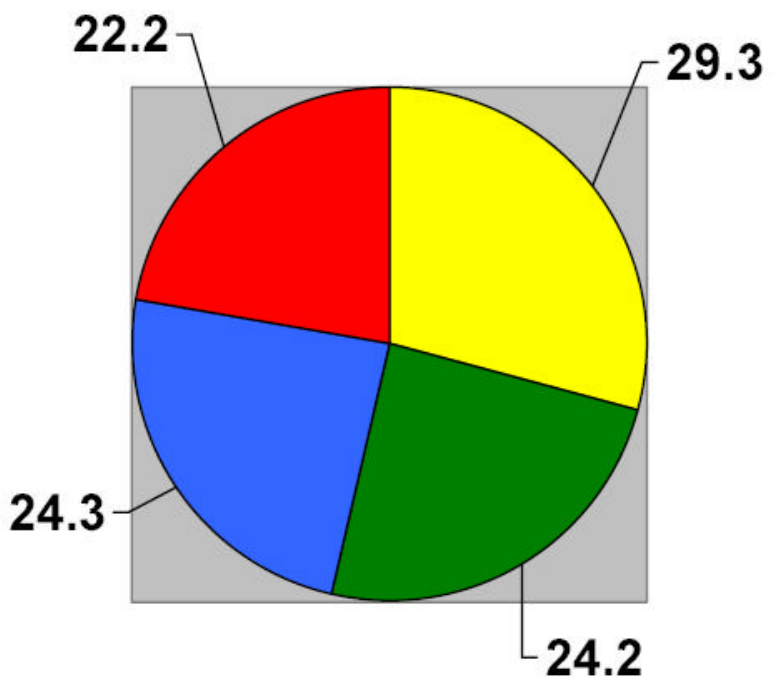

\begin{tabular}{|l|}
$\square$ Route \\
$\square$ Vertebral Fractures \\
$\square$ Hip Fractures \\
$\square$ Adverse Effects
\end{tabular}

Figure 1.

Relative Importance of Specific Medication Characteristics*

* Values sum to 100 


\section{Table 1}

Participant Characteristics

\begin{tabular}{lc}
\hline Characteristic & Number $(\mathbf{\%})(\mathbf{T o t a l}=\mathbf{2 1 2})$ \\
\hline Age (median, range) & $73(46-90)$ \\
Women & $201(95)$ \\
Caucasian & $185(87)$ \\
Married & $106(50)$ \\
At least some college education & $143(68)$ \\
High Fracture Index & $81(38)$ \\
High perceived risk of fracture & $55(26)$ \\
Currently using bisphosphonates & $120(57)$ \\
Currently using rhPTH & 1 \\
Health status very good or excellent & $102(48)$ \\
Previous vertebral fracture & $20(9)$ \\
Previous hip fracture & $5(2)$ \\
Preferring to treat health problems without doctors or prescription drugs $(\%)$ & $37(18)$ \\
\end{tabular}




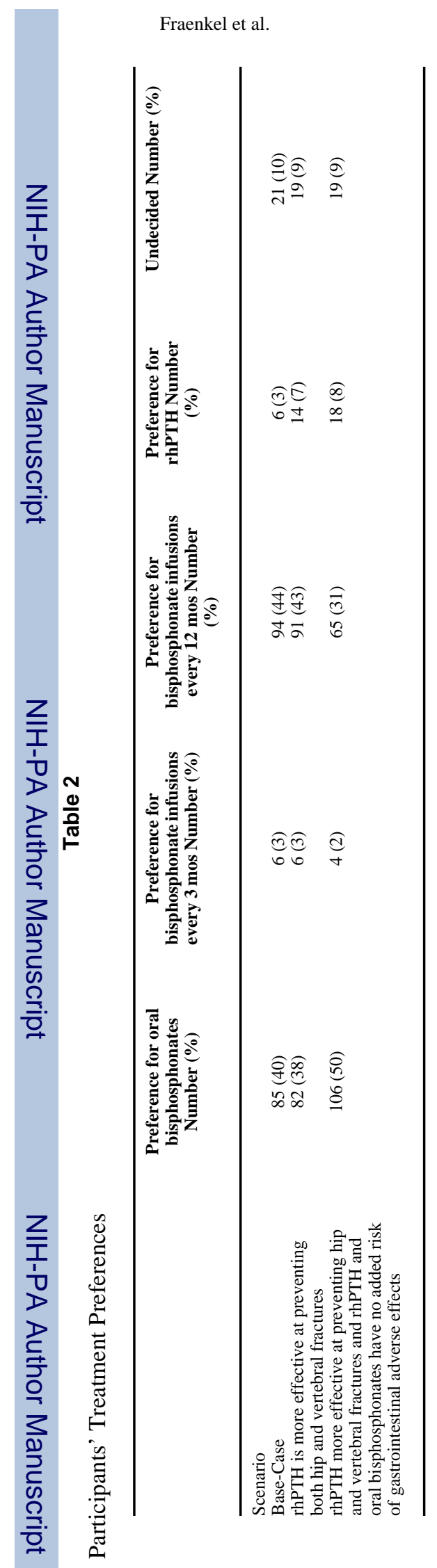

Arthritis Rheum. Author manuscript; available in PMC 2006 October 26. 
Table 4

Associations between Participants' Characteristics and Treatment Preference*

\begin{tabular}{|c|c|c|c|c|}
\hline Characteristic & $\begin{array}{c}\text { Prefer Oral } \\
\text { Bisphosphonates } \\
\text { (Number = 28) }\end{array}$ & $\begin{array}{c}\text { Prefer IV } \\
\text { Bisphosphonates } \\
\text { (Number = 52) }\end{array}$ & $P$ value & $\begin{array}{l}\text { Adjusted Odds } \\
\text { Ratio }(95 \% \text { CI })^{* *}\end{array}$ \\
\hline Age $\geq 65(\%)$ & 86 & 75 & 0.3 & - \\
\hline Some college education $(\%)$ & 61 & 62 & 0.9 & - \\
\hline Has prescription drug plan (\%) & 54 & 69 & 0.2 & - \\
\hline $\begin{array}{l}\text { Health status very good or } \\
\text { excellent }(\%)\end{array}$ & 64 & 37 & 0.02 & $3.0(1.0-8.5)$ \\
\hline High perceived risk of fracture $(\%)$ & 7 & 31 & 0.02 & $0.2(0.04-1.2)$ \\
\hline $\begin{array}{l}\text { Preferring to treat health problems } \\
\text { without doctors or prescription } \\
\text { drugs }(\%)\end{array}$ & 7 & 31 & 0.02 & $0.1(0.03-0.7)$ \\
\hline
\end{tabular}

